\title{
Aggressive periodontitis association with recessive hyperkeratotic mutation (a case report of 3 siblings)
}

\begin{abstract}
Specific systemic disorders increase the patient's susceptibility to periodontal disease, either by compromising the immune response or by influencing the mineralization of bone and dental tissues, in consequence, patients affected with those illnesses may additionally deteriorate to severe periodontitis and typically lose their teeth at an early age. Systemic diseases with a periodontal element encompass rare genetic syndromes in which the patients expand aggressive periodontitis as a secondary manifestation. In this report, the clinical presentation, differential diagnosis, comprehensive and periodontal status of three siblings affected by aggressive periodontitis in association with recessive hyperkeratotic mutation is discussed.
\end{abstract}

Keywords: papillon-lefèvre syndrome, haim-munk syndrome, aggressive periodontitis
Volume 9 Issue 4 - 2018

\author{
Ahmed Tawfig \\ Department of Dentistry, Riyadh Elm University, Saudi Arabia
}

Correspondence: Ahmed Tawfig, Assistant Professor,

Department of Dentistry, Riyadh Elm University, King Fahad

Road, Riyadh, Saudi Arabia, Tel 009665642 I 3932

Email ahmed.tawfig@riyadh.edu.sa

Received: July 27, 2018 | Published: August 09, 2018

\section{Background}

Specific systemic disorders increase the patient's susceptibility to periodontal disease, either by compromising the immune response (particularly neutrophil count and function) or by influencing the mineralization of bone and dental tissues ${ }^{1}$ in consequence, patients affected with those illnesses may additionally deteriorate to severe periodontitis and typically lose their teeth at an early age. Systemic diseases with a periodontal element encompass rare genetic syndromes in which the patients expand aggressive periodontitis as a secondary manifestation. ${ }^{1}$ Management of the periodontal elements of these diseases could be challenging. Proper diagnosis is mandatory for a proper control; as a result, a differential diagnosis needs to be entrenched and the related genetic disease identified if achievable. Papillon-Lefèvre syndrome (PLS) is a rare autosomal recessive heterogeneous disorder, which is characterised by palmoplantar hyperkeratosis, early loss of primary and permanent teeth, ${ }^{2}$ has a worldwide prevalence of 1-4 cases per million in the general population and is often related with consanguinity. ${ }^{3}$ The onset of the disease usually coincides with the eruption of primary teeth. Boys and girls are equally affected, with no racial predominance ${ }^{4}$ Specific Palmoplantar keratoderma (PPK) forms can be recognised by prominent mucosal involvement. In particular, severe periodontitis with premature tooth decay typifies two peculiar PPKs due to mutations of the cathepsin C (CTSC) gene..$^{5-8}$ The Haim-Munk (HMS) and the Papillon-Lefevre (PLS) syndromes. ${ }^{3,9}$ Cathepsin $\mathrm{C}$ is a lysosomal cysteine protease acting as an activator of serine proteases of immune and inflammatory cells ${ }^{7}$ and possibly as a modulator of epithelial differentiation. ${ }^{10}$

Palmoplantar keratoderma with periodontitis due to CTSC mutation (Haim-Munk syndrome) HMS was first described by Haim \& Munk ${ }^{11}$ in 1965 in members of a small Jewish community in India. It is a rare autosomal recessive disorder due to CTSC mutations. ${ }^{12}$ HMS manifests in early childhood with diffuse or focal (frictionrelated) hyperkeratosis that frequently extends on back aspects of hands and feet, lateral malleoli and Achilles' tendons, elbows and knees. Severe and early-onset periodontitis causes premature loss of deciduous and permanent teeth. Recurrent skin infections are frequent additional findings. Onychogryphosis, arachnodactyly, acroosteolysis, pes planus (flatfoot) and permanent flexion contractures are pathognomonic of HMS., ${ }^{5,6,12}$ Palmoplantar keratoderma with periodontitis due to CTSC mutation (Papillon-Lefevre syndrome)
PLS is a rare allelic disorder also due to recessive mutations in CTSC. 25,28 It usually manifests in early childhood and shares with HMS several ${ }^{3,10}$ clinical features, such as diffuse or focal (friction-related) hyperkeratosis, severe periodontitis with premature loss of deciduous/ permanent teeth and recurrent skin infections. Hyperkeratosis may be less severe than in HMS, whereas the periodontium is more severely affected $^{6,12}$ Dural and choroid plexus calcifications and mild mental retardation may be present. Abscess involving internal organs such as liver, kidney, lung and brain as well as high serum IgE levels is described. ${ }^{7,13}$ Diagnosis is principally based on careful clinical evaluation (Figure 1). Positive family history and onset in infancy or childhood are relevant to the distinction between hereditary and acquired PPK, the latter usually showing negative family history and later onset. ${ }^{14}$ Diffuse, focal or punctate manifestations and the presence of disfigurements are significant findings in dermatological diagnosis. Although PPK is not life-threatening, it strongly impairs patient quality of life by reducing acral functionality and causing discomfort and pain. Currently, available treatments for PPK are not curative but only aimed at symptom-relieving. Daily bathing and professional foot and hand care are helpful for mechanical scale removal (e.g. with pumice stone) in some PPK types. Regular application of emollients (white petrolatum), keratolytic (urea, salicylic acid) and wet dressings may be used to control hyperkeratosis. Topical corticosteroids are used to reduce inflammation, when present. Hyperhidrosis may respond to aluminium chloride containing products. Local infections should be treated with antifungal and antibacterial drugs. Iontophoresis and botulinum toxin has been reported to improve symptoms of PPK caused or exacerbated by water exposure. ${ }^{15}$

\section{Case reports}

In this report, the clinical presentation, differential diagnosis, comprehensive and periodontal status of three siblings with PLS is discussed. They were born of none consanguineous healthy parents; the father has 12 brothers and sisters were the $4^{\text {th }}$ brother or sister is always affected by the same disease. Two sisters and their brother attended our periodontal clinic at Riyadh Elm University with their mother seeking urgent treatment for the elder sister complaining of dental abscess and loss of teeth, direct extra and intraoral examination revealed the need to go for comprehensive examination for the brother and sisters because suspected PPS 


\section{Case one}

A (10 years) old girl presented with multiple early teeth loss and delayed eruption of the permanent dentition. Her mother mentioned that there were eruptions on the scalp, legs, feet, arms, and hands at birth, for which the parents did not seek any treatment. Subsequently, there was reddening of the palms and soles at six months of age, which gradually thickened and became rough and scaly. These symptoms were worse during the winter season, her teeth started to be loose and mobile till she lost her primary teeth at the age of two; intraoral examination reveals missing all the deciduous teeth in the upper and lower jaw with the existence of only the imcisors and first molars in all quadrants, Patient had a reduced facial height due to resorption of the alveolar ridge. General examination, the patient had a weak physique, the physical and mental development was regular. Dermatological examination revealed increased keratinization of the skin of the palmar and plantar surfaces as well as the skin over the dorsal surfaces of the hand wrists ; the keratinized skin was demarcated from the adjacent healthy skin. Deep fissures were present on the soles of feet. Her nails and hair were regular (Figure 1)
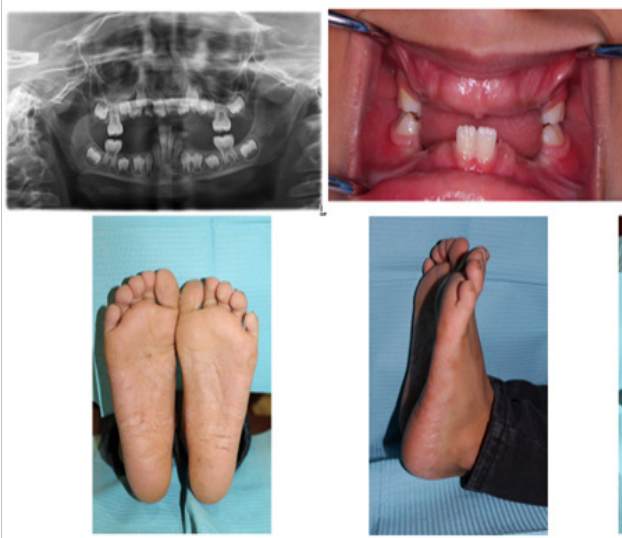
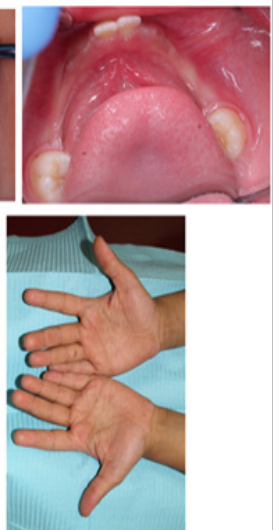

\section{Case two}

A (14 years) old girl complained of multiple dental abscess with pus coming out from the gingival sulcus simultaneously, slight calculus detected also and moderate gingivitis in association with floating and mobility in all the present teeth. The mother gave exactly the same natal history of the dermatological and dental status of her younger sister. While extraoral examination reveals, worsened skin features. The radiographs showed an aggressive form of bone destruction around her upper and lower incisors and the first molars (Figure 2).
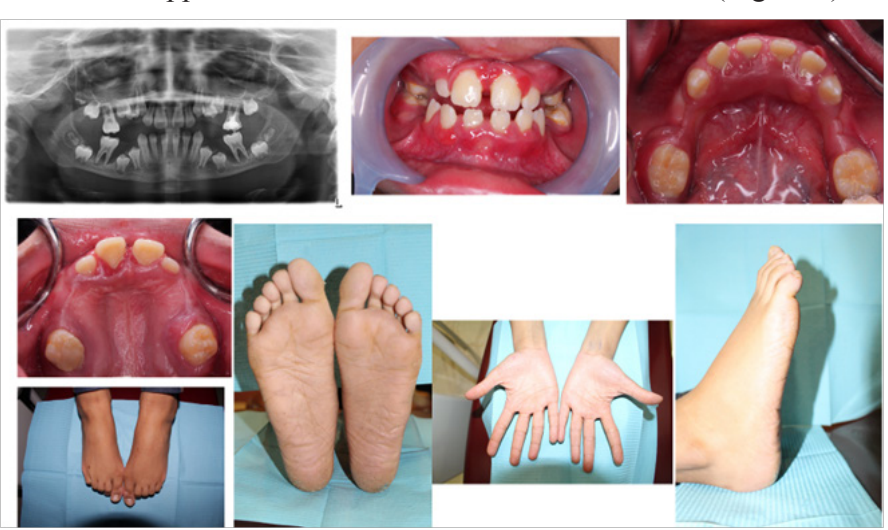

\section{Case three}

A (16 years) old boy with regular dental status except for multiple carious teeth and extracted 25 and 35 with also generalized mild gingivitis. The radiographs revealed standard bone structure and levels. The natal history taken from the mother showed the same features for the dental and dermatological status of his sisters, but his secondary teeth erupted normally. Also, she mentioned that the dermatological status started to be severe at the age of puberty, they tried to seek treatment, but nothing corrected his condition. Extraoral examination showed a severe form of keratosis in the joints and extremities with deep fissures in the palms and feet. Also, his nails were curved, and his fingers were taller than usual with (flatfeet) showed clearly (Figure 3).

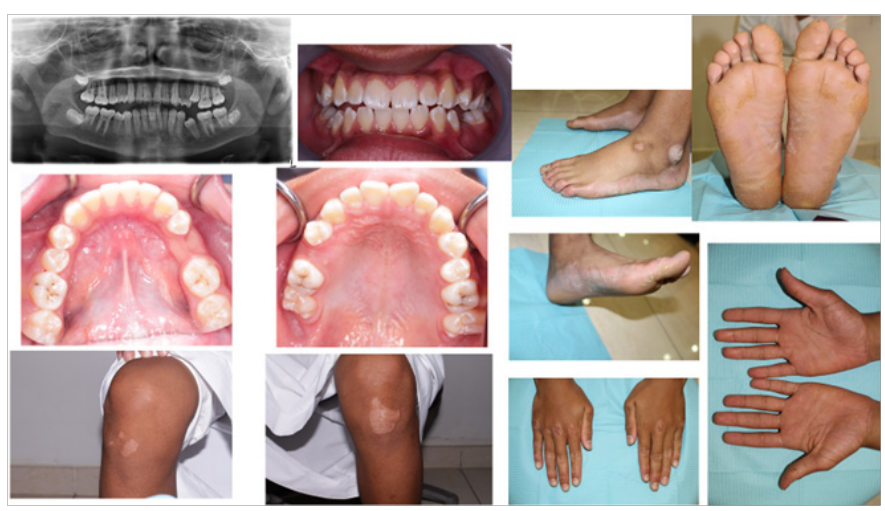

Laboratory investigation carried out, which included a haematological and biochemical valuation. The results were within standard parameters. Considering of the above findings, the cases were differentially diagnosed as either Palmer Plantar Syndrome (PLS) or Hiam-Munk syndrome.

\section{Discussion}

Because of the low economic status of the parents, genetic testing could not be performed to identify the gene mutation, but the periodontal, dermatological and radiological features strongly proposed the diagnosis of PLS. Moreover, the hereditary descent has been described for this syndrome, and the three cases reported here were also associated with consanguinity of parents. The parents were healthy, and there was a family history of the disease, suggesting an autosomal recessive pattern of inheritance. The differential diagnoses include Hiam-Munk syndrome. (HMS) Also exhibits arachnodactly, acroosteolysis, atrophy of nails, and deformity of the phalanges in the hands. however, the case of the eldest brother with severe dermatological features may give the possibility of Hiam-Munk syndrome. PLS can adversely affect growing children psychologically, socially, and aesthetically. Hence, early dental evaluation and parental counselling as a part of preventive dental treatment are essential for providing complete psychosocial rehabilitation for PLS children; a multidisciplinary approach may improve the prognosis and quality of life of these children. A final treatment regime is not yet conveyed; however, to control periodontal destruction, several treatment modalities have been suggested, e.g., conventional periodontal therapy, oral hygiene instructions, and systemic antibiotics. Further research is essential for designing a treatment strategy that can save the smiles of these children. 


\section{Conclusion}

The occurrence of aggressive periodontitis in association with recessive hyperkeratotic mutation among siblings is documented, although this is the first case where Papillon-Lefevre syndrome and Hiam-Munk syndrome affect the three siblings of the same family. There is no definitive treatment for PLS neither HMS cases. Symptomatic management is followed. A multidisciplinary tactic with the active contribution of a periodontist, pediatrician and dermatologist is essential for the management of case of aggressive periodontitis association with recessive hyperkeratotic mutation.

\section{Acknowledgements}

None.

\section{Conflict of interest}

The author declares that there is no conflict of interest.

\section{References}

1. Albandar JM. Aggressive and acute periodontal diseases. Periodontol 2000. 2014;65(1):7-12.

2. Gorlin RJ, Sedano H, Anderson VE. The syndrome of palmar-plantar hyperkeratosis and premature periodontal destruction of the teeth. A clinical and genetic analysis of the papillon-lefevre syndrome. J Pediatr. 1964;65(6):895-908.

3. Hart TC, Hart PS, Bowden DW, et al. Mutations of the cathepsin C gene are responsible for Papillon-Lefèvre syndrome. Journal of Medical Genetics. 1999;36(12):881-887.

4. Khan F, Jan S, Mushtaq M. Papillon-Lefevre syndrome: Case report and review of the literature. J Indian Soc Periodontol. 2012;16(2):261-265.

5. Aswath N, Swamikannu B, Ramakrishnan S, et al. Heterozygous Ile453Val codon mutation in exon 7, homozygous single nucleotide polymorphisms in intron 2 and 5 of cathepsin $\mathrm{C}$ are associated with Haim-Munk syndrome. Eur J Dent. 2014;8(1):79-84.
6. Janjua SA, Iftikhar N, Hussain I, et al. Dermatologic, periodontal, and skeletal manifestations of Haim-Munk syndrome in two siblings. Journal of the American Academy of Dermatology. 2008;58(2):339-344.

7. Nikoletta N, Péter V, Zsanett C, et al. CTSC and Papillon-Lefèvre syndrome: detection of recurrent mutations in Hungarian patients, a review of published variants and database update. Mol Genet Genomic Med. 2014;2(3):217-228.

8. Romero-Quintana JG, Frías-Castro LO, Arámbula-Meraz E, et al. Identification of novel mutation in cathepsin $\mathrm{C}$ gene causing PapillonLefèvre Syndrome in Mexican patients. BMC Medical Genetics. 2013;14(1):7.

9. Reena R, Thiagarajan S, Soumya Mohandas. Haim Munk syndrome and Papillon Lefevre syndrome-allelic mutations in cathepsin C with variation in phenotype. International Journal of Dermatology. 2010;49(5):541-543.

10. Toomes C, James J, Wood AJ, et al. Loss-of-function mutations in the cathepsin $\mathrm{C}$ gene result in periodontal disease and palmoplantar keratosis. Nat Genet. 1999;23(4):421-424.

11. Haim S, Munk J. Keratosis Palmo-Plantaris Congenita, With PerioDontosis, Arachnodactyly and a Peculiar Deformity of the Terminal Phalanges. Br J Dermatol. 1965;77:42-54.

12. Hart TC, Hart PS, Michalec MD, et al. Haim-Munk syndrome and Papillon-Lefèvre syndrome are allelic mutations in cathepsin C. J Med Genet. 2000;37(2):88-94.

13. LiZ, Liu J, Fang S, et al. Novel compound heterozygous mutations in CTSC gene cause Papillon-Lefèvre syndrome with high serum immunoglobulin E. Journal of Dermatological Science. 2014;76(3):258-260.

14. Patel S, Zirwas M, English JC. Acquired Palmoplantar Keratoderma. Am J Clin Dermatol. 2007;8(1):1-11.

15. Diba VC, Cormack GC, Burrows NP. Botulinum toxin is helpful in aquagenic palmoplantar keratoderma. British Journal of Dermatology. 2005;152(2):394-395. 